\title{
Interaction between anions and substituted molecular bowls
}

Enrique M. Cabaleiro-Lago ${ }^{(a)}$, Jesús Rodríguez-Otero ${ }^{\left({ }^{b}\right)}$ Ángeles Peña-Gallego ${ }^{\left({ }^{b}\right)}$

(a) Departamento de Química Física, Facultade de Ciencias, Universidade de Santiago de Compostela, Campus de Lugo. Avda. Alfonso X El Sabio s/n 27002 Lugo, Galicia (Spain).

(b) Departamento de Química Física, Facultade de Química, Universidade de Santiago de Compostela, Avda. das Ciencias, s/n 15706 Santiago de Compostela, Galicia (Spain).

\section{e-mail: caba.lago@usc.es}

\begin{abstract}
Complexes formed by anions and substituted molecular bowls were studied by means of computational calculations with density functional theory methods. An empirical dispersion term was included to correct the well known flaws of common functionals to describe dispersion interactions. The modified bowls consisted of corannulene molecules substituted with five or ten F, $\mathrm{Cl}$, or $\mathrm{CN}$ units, whereas $\mathrm{Cl}^{-}, \mathrm{Br}^{-}$and $\mathrm{BF}_{4}^{-}$where the anions considered.
\end{abstract}

Substitution with $\mathrm{F}, \mathrm{Cl}$ and $\mathrm{CN}$ produces an inversion of the molecular electrostatic potential of the bowls, which become positive over the two faces of the bowl, therefore interacting favorably with anions.

Three different structures were found for each of the complexes, corresponding to one arrangement with the anion interacting with the concave side of the bowl and two different arrangements with the anions interacting with the convex side of the bowl.

The strength of the interaction roughly follows the values of molecular electrostatic potential, being more stable as more positive is the potential. However, the role of dispersion arises as essential for having a correct order of stabilities. In fact, when dispersion is included, most complexes are more stable by the concave face, where the closer proximity of the atoms allows for a larger dispersion effect. Also, it is dispersion which makes the complexes formed with a larger anion as $\mathrm{BF}_{4}^{-}$as stable as those formed with $\mathrm{Cl}^{-}$or $\mathrm{Br}^{-}$. 
The results suggest the possibility of employing these substituted molecular bowls as receptors for anions with a preferential concave complexation, specially for structured anions where dispersion effects will be larger. 


\section{Introduction}

Intermolecular interactions involving aromatic rings play important roles in many areas in chemistry from materials design to molecular biology. The interactions are important for structures of molecular crystals, stability of biological systems and their molecular recognition processes. ${ }^{1,2}$ The study of the fundamentals of these interactions is important for helping the design of new materials as well as for understanding cluster formation processes. Over the past decade, novel types of interaction involving aromatic rings have been an important subject. In this regard, when the interaction implies an aromatic unit it is usually one of the following three types: cation $\cdots$,

$\cdots$ or $\mathrm{XH}^{\cdots}$, though new possibilities as the anion $\cdots$ interaction have been considered in recent years. ${ }^{3-5}$

The properties of aromatic polycyclic compounds, and more specifically polycyclic aromatic hydrocarbons $(\mathrm{PAH})$ have attracted much interest regarding possible applications in materials science. A deep knowledge of the interaction with these systems is important for understanding the behaviour of nanotubes or fullerenes in view of their potential applications. Therefore, a thorough understanding of the interactions involving these species is crucial for different applications as also is for developing appropriate force fields leading to more realistic simulations in complex systems.

During the last years, an interest has aroused with respect to the characteristics of aromatic systems presenting curved surfaces, which therefore lead to different properties depending on the face considered. Klärner showed that for certain molecular tweezers, the electrostatic potential is significantly more negative inside the tweezer than for the outer face, so it could be possible to employ such systems as appropriate receptors for electron deficient molecules. ${ }^{6}$

This kind of behaviour was not only observed in molecular tweezers as those proposed by Klärner. Also, molecular bowls exhibit differences in the electrostatic potential depending on whether the concave of convex face is considered. ${ }^{6,7}$

These molecular bowls are aromatic systems formed by joining six and five carbon rings in a similar way as in fullerenes, the five carbon rings introducing curvature on the delocalized system. For the simplest of these bowls, corannulene, $\mathrm{C}_{20} \mathrm{H}_{10}$, the electrostatic potential is different depending on whether the concave or the convex side of the bowl is considered and, in fact, alkaline cations are known to preferentially bind corannulene by the convex face. ${ }^{7,8}$ 
Recently, it has also aroused interest the possibility of stabilizing interactions between anions and electron deficient aromatic systems in the so-called anion $\cdots \pi$ interaction. The extensive work of Fradera et al. has already shown that the interaction is mainly electrostatic in nature, so one must have an electron deficient aromatic system with positive quadrupole moment, such as hexafluorobenzene of triazine. The attractive interaction between this quadrupole and the negative charge of the anion is mainly responsible of the stabilizing interaction, with strengths similar to those observed for cation $\cdots \pi$ interactions. ${ }^{5,9,10}$

Most studies in this field have been carried out employing benzene as a model for the aromatic system, so these works have mostly considered hexafluorobenzene, trifluorobenzene or triazine complexes with halogen anions. ${ }^{5,9-11}$ However there is a lack of studies of the interaction with more complex anions or with more extended aromatic systems. Recently, Hermida-Ramón et al. ${ }^{12}$ have performed a study of the interaction of iodine anion with a perfluorinated molecular tweezer showing a significant interaction, thus suggesting the possibility of complexation of the anion by the tweezer.

It can be expected that for more extended aromatic systems, the contribution of inductive forces will be larger than in benzene derivatives. Also, as indicated by Kim et al., the anion $\cdots \pi$ interaction presents a more dispersive character than the cation $\cdots \pi$ contact, so in complexes formed by large aromatic systems and more complex anions the dispersion contribution could be significant.

The measurement of the interaction between polar systems is usually quite straightforward, but it is much more difficult to measure the magnitude of the interaction when dispersion forces play an important role. The interaction in complexes involving aromatic moieties is only accurately described by employing the most rigorous methods, mainly due to the large correlation/dispersive component of the interaction. ${ }^{4}$ This means that only medium size systems can be studied with such a degree of sophistication, larger PAHs being computationally out of the scope for those methods.

Common DFT functionals are unable to describe the interaction between aromatic systems properly, usually predicting non-bonded situations. Though the interaction with anions should be more easily described due to its electrostatic character (at least its main features), it can be expected that the dispersive component will be lost by using common functionals. Very recently, following an old idea applied to the HF method, Grimme proposed adding an empirical term to several common 
functionals as an easy and unexpensive way of including dispersion, in the so called DFT-D approach. ${ }^{13,14}$ Doing so, the performance of commonly used functionals is greatly improved without extra computational costs. Other approaches trying to give good descriptions of the intermolecular interaction at a moderate computational cost include new designed functionals ${ }^{15,16}$ or empirical scaling of the MP2 method. ${ }^{17,18}$

In the present work, a computational study of complexes formed by several anions and molecular bowls is presented. The systems thus studied are presented in Figure 1. The molecular bowls are constructed from corannulene by substituting several hydrogen atoms by chlorine, fluorine or nitrile groups, pretending to produce an inversion of the molecular electrostatic potential (MEP) thus allowing a stabilising interaction with anions. To the best of our knowledge only the chlorinated derivatives have been already synthetised to date. ${ }^{8}$ As anions, chlorine and bromine were employed as representative of the simplest halogen anions. Fluorine was not considered since it binds to the molecular bowl in an almost covalent fashion. Finally, a more structured anion as $\mathrm{BF}_{4}^{-}$was employed to estimate the effect of including larger anions over the dispersive component of the interaction.

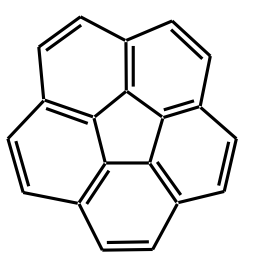

corannulene
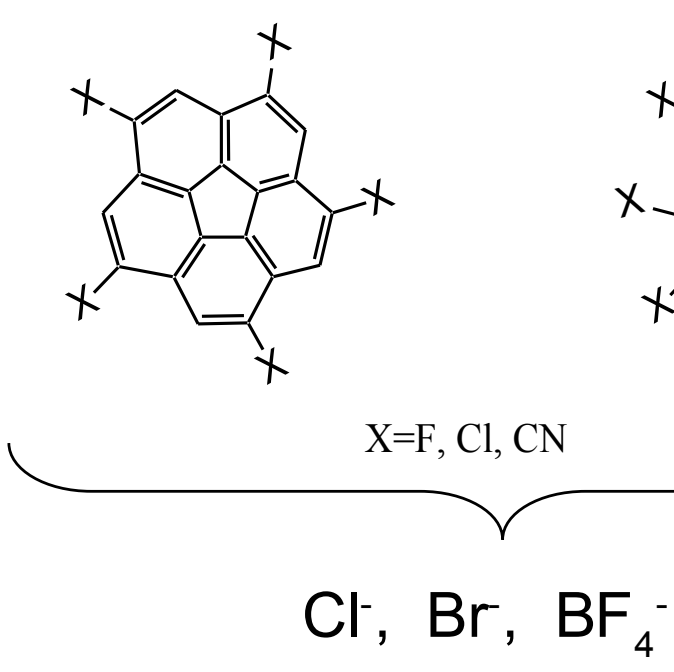

Figure 1. Molecular Bowls and anions employed in the present study. 
By studying these systems information can be obtained regarding the characteristics of the interaction of anions with an extended curved $\pi$ system. By comparing the different bowls it would be possible to asses the effect produced by changing the molecular electrostatic potential of the bowl over the strength of the interaction. Also, studying the systems indicated in Figure 1, information about the preference of concave/convex complexation and of the role of dispersion interactions in this kind of systems will be obtained.

\section{Computational Details}

Clusters formed by the substituted corannulenes and the anions indicated in Figure 1 were computationally studied by using density functional theory. Starting structures were constructed by placing each of the anions over the different hexagonal or pentagonal faces of the substituted corannulenes, both in the concave and the convex sides. These initial structures were fully optimized employing the BLYP functional corrected by an empirical dispersion term as designed by Grimme (BLYP-D). ${ }^{13,14}$ The reason of employing a dispersion corrected functional comes from the possibility of dispersion being more important than in cation $\cdots \pi$ interactions. It can be expected that the combination of a larger, curved system, together with the presence of anions, will lead to a larger contribution of dispersion which should be almost completely lost with common functionals.

BLYP-D was chosen since in previous work has shown to produce pretty good results for the interaction with polycyclic aromatic species, providing a good description of intermolecular interactions involving $\pi$ systems. ${ }^{19}$ Besides, with a non-hybrid functional, computational effort can be saved by applying the resolution of the identity (RI) approach.

The structures were initially optimized with the TZVP basis set together with the TZVP auxiliary basis set for RI calculations. Since this basis set does not include diffuse functions and therefore it cannot be expected to provide a reliable description of anion interactions, structures were reoptimized by using the aug-cc-pVDZ basis set, again with the TZVP auxiliary basis set.

After locating the stationary points of the potential energy surface of each cluster, the interaction energies were calculated by means of the counterpoise method to avoid basis set superposition error. $^{20,21}$ Thus, the interaction energy results from subtracting the energies of the fragments that constitute the clusters employing the geometry and the whole basis set of the cluster 


$$
\Delta E_{\text {int. }}=E_{i j}(i j \ldots)-\sum_{i} E_{i j}^{\text {clus. }}(i j \ldots)
$$

As the geometry of the molecules changes when the cluster is formed, and additional contribution describing this effect must be included, obtained as the energy difference between the molecules in the cluster geometry and in isolation.

$$
E_{d e f}=\sum_{i}\left[E_{i}^{\text {clus. }}(i)-E_{i}^{\text {isol. }}(i)\right]
$$

The total complexation energy results from adding these two contributions, though deformation effects are usually small and negligible for most clusters, and in the discussion we will not consider them. All calculations were performed with the Orca and Turbomole programs. ${ }^{22,23}$ 

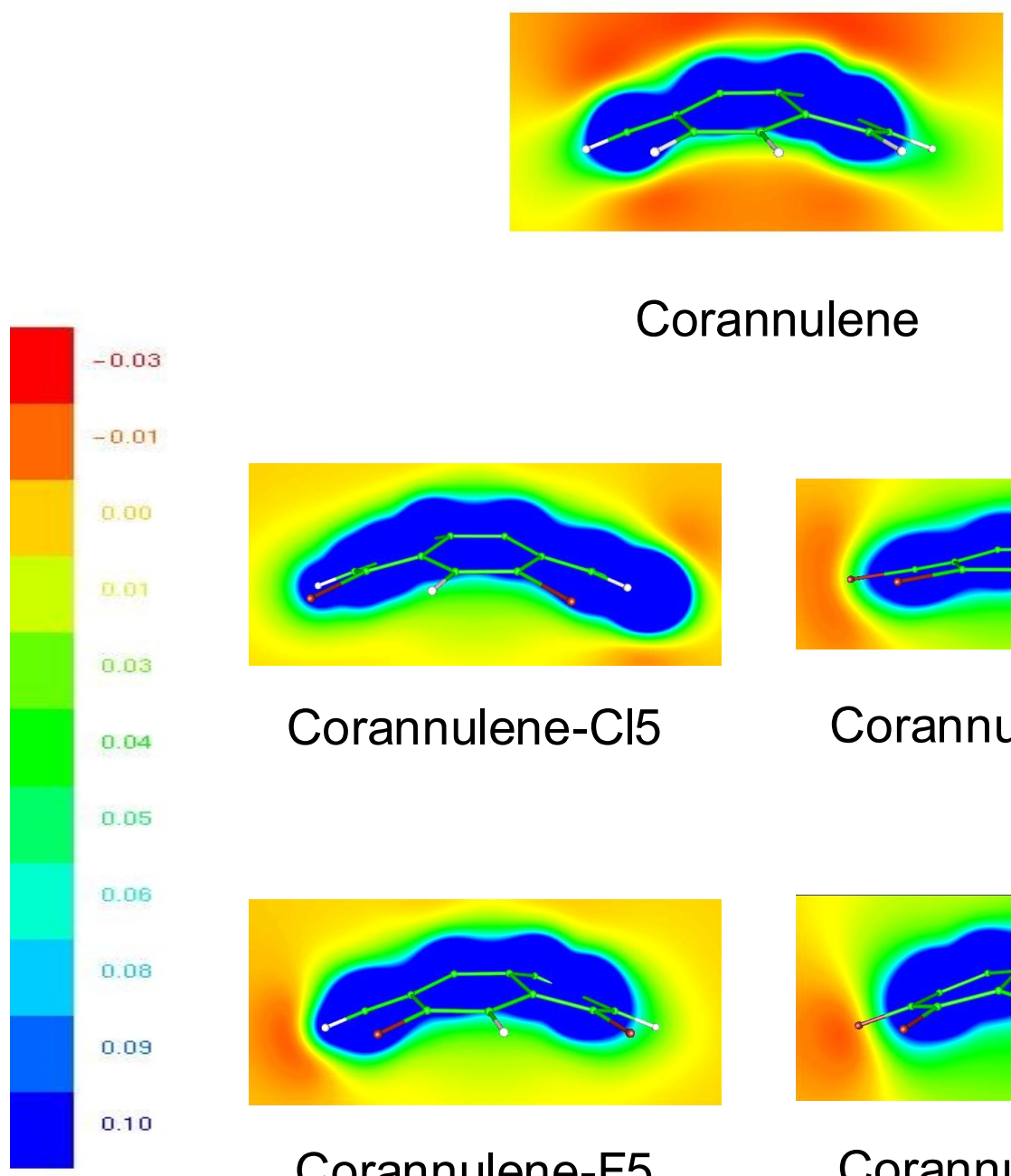

Corannulene

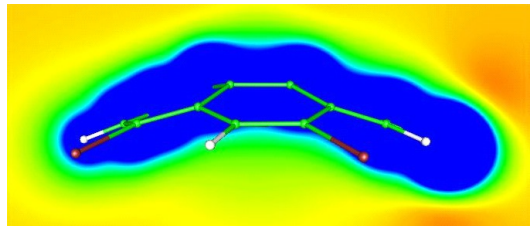

Corannulene-Cl5

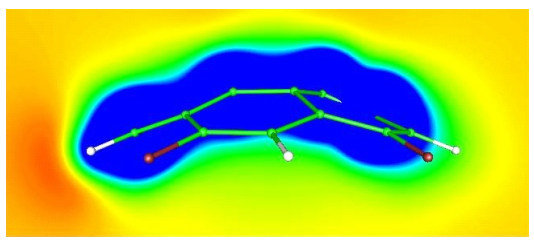

Corannulene-F5

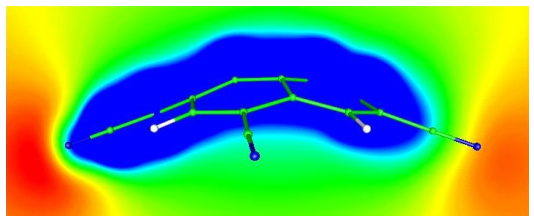

Corannulene-CN5

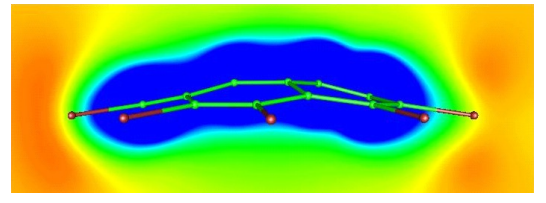

Corannulene-Cl10

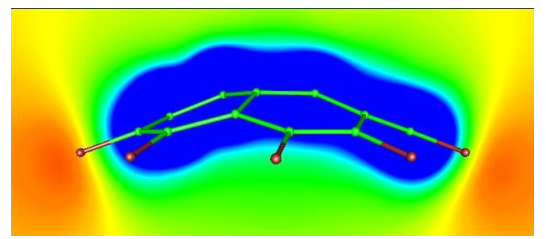

Corannulene-F10

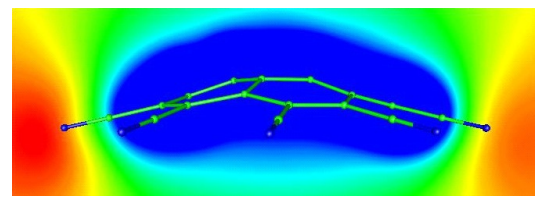

Corannulene-CN10

Figure 2. Molecular electrostatic potential of the molecular bowls employed in this study obtained at the BLYP/aug-cc-pVDZ level of calculation. The scale is shown in atomic units. 


\section{Results}

\subsection{Substituted Molecular Bowls}

Figure 2 shows the molecular electrostatic potential maps for the substituted molecular bowls studied in the present work, together with the original corannulene molecule as reference. The molecular electrostatic potential is represented onto a plane containing the $\mathrm{C}_{5}$ symmetry axis of the molecule and cutting the pentagonal ring perpendicularly to stress the differences between the convex and the concave sides of the molecular bowl.

It can be observed that for corannulene the convex face presents at this level of calculation a more negative electrostatic potential than the concave face, but in both sides the MEP is negative. Therefore one can expect cations to bind preferentially, in an electrostatic basis, to the convex side of the molecule, as shown in previous work for alkaline cations. ${ }^{7}$

As electron withdrawing substituents are included in the molecular bowl, the MEP's become more positive. In fact, for all the molecular bowls studied in this work, the MEP's are positive in both faces of the bowl. In any case, it is easy to see that the MEP becomes more positive in the order $\mathrm{CN}>\mathrm{F}>\mathrm{Cl}$. Also, decasubstituted bowls present more positive MEP's than the corresponding pentasubstituted ones. It is also worth noting that substitution with $\mathrm{CN}$ produces the largest effect, giving raise to the most positive electrostatic potentials.

These changes can be observed more clearly in Figure 3, where the molecular electrostatic potential is represented along the $\mathrm{C}_{5}$ axis of the molecular bowls.

It can be observed that only for the original corannulene molecule negative electrostatic potential regions are obtained, being more negative in the convex face of the bowl. Introducing chlorine substituents in the molecule gives MEP's that are slightly positive along the axis, and the same occurs when the molecular bowl is fluorinated. As regards MEP, the effect of introducing five chlorine or fluorine substituents is similar. When ten substituents are introduced, the MEP's become even more positive, and more differences are observed between fluorine and chlorine derivatives, specially in the concave face. 
The most striking effect is produced by substitution with $\mathrm{CN}$ groups. Already with $5 \mathrm{CN}$ groups in the molecule, the MEP becomes much more positive than any of the molecules including $\mathrm{F}$ or $\mathrm{Cl}$. Again, the effect is much larger when $10 \mathrm{CN}$ groups are introduced. Taking into account the results shown in Figures 2 and 3, it can be expected that complexes formed with the derivatives containing $\mathrm{CN}$ groups will be the most stable by far, whereas complexes formed with fluorinated or chlorinated bowls will roughly present similar interaction energies.

Table 1. Selected geometric parameters of the molecular bowls studied.

\begin{tabular}{|l|c|c|l|c|c|}
\hline & $\mathbf{R}_{\text {bottom }}(\AA)$ & $\mathbf{R}_{\text {extreme }}(\AA)$ & & $\mathbf{R}_{\text {bottom }}(\AA)$ & $\mathbf{R}_{\text {extreme }}(\AA)$ \\
\hline Corannulene & 0.940 & 6.522 & & & \\
\hline C15 & 0.923 & 6.521 & $\mathbf{C l 1 0}$ & 0.553 & 6.699 \\
\hline F5 & 0.933 & 6.505 & F10 & 0.889 & 6.524 \\
\hline CN5 & 0.924 & 6.539 & $\mathbf{C N 1 0}$ & 0.731 & 6.649 \\
\hline
\end{tabular}

As a way of checking the changes introduced in the bowls by the substituents Table 1, lists the values of two characteristic distances: the distance from the rim carbon atoms to the plane of the five-membered ring, and the longest distance between two carbon atoms of the rim of the bowl.

It can be observed that incorporation of the substituents gives more planar bowls. This effect is smaller for the pentasubstituted bowls, and almost negligible for the fluorinated derivative. However, in the case of the decasubstituted derivatives the effect is much larger, specially in the chlorinated compound, which is the more planar bowl among those studied in this work. This greater planarity will affect the interaction with the different anions, and will reduce the differences between the convex and concave sides. It can be expected that dispersion interactions will favor complexation by the concave face, where more atoms can be in closer proximity to the anion. 


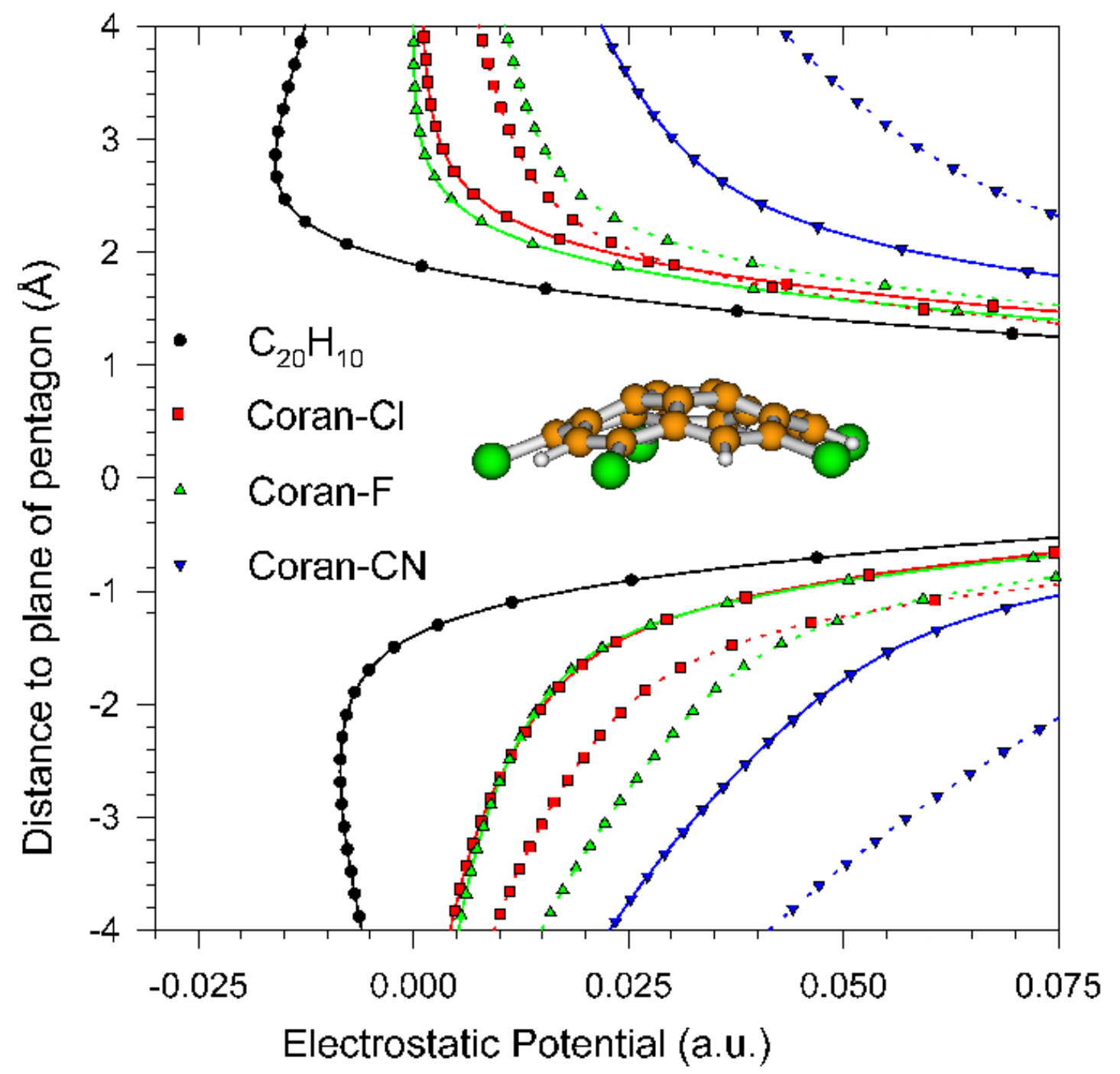

Figure 3. Molecular electrostatic potential of the molecular bowls studied in this work. The figure represents the MEP's along the $\mathrm{C}_{5}$ symmetry axis of the bowls, as obtained at the B-LYP/aug-cc-pVDZ level of calculation. Top: convex side. Bottom: concave side. Dotted line: 10 substituents. Solid line: 5 substituents. 

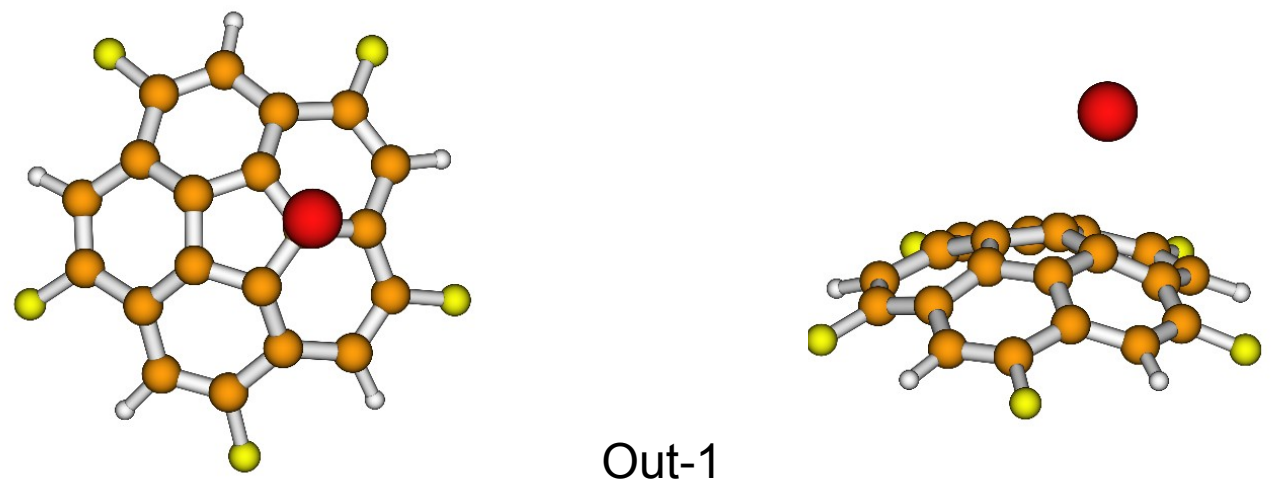

Out-1
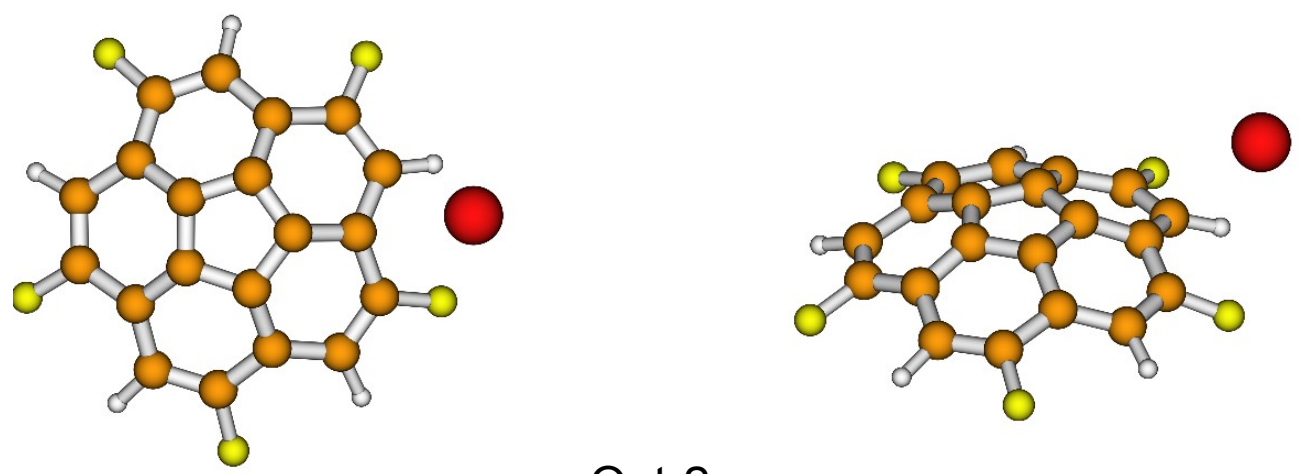

Out-2
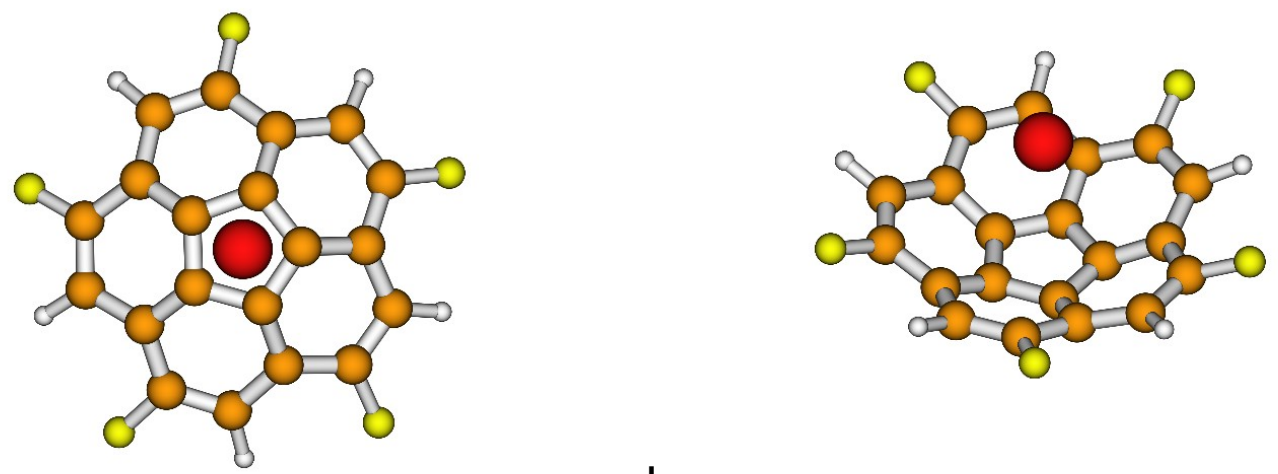

In

Figure 4. Minimum energy structures obtained for the complexes formed by bromide and Coran-F5. For the other bowls and anions similar structures were also found. 


\subsection{Complexes between anions and molecular bowls}

Figure 4 shows the optimized structures of the complexes formed by bromide and the pentafluorinated bowls. The geometrical arrangements found with other bowls or anions present similar characteristics. In all cases, three different arrangements were obtained. One of the structures corresponds to a disposition where the anion is located by the concave face of the bowl (In), located over the symmetry axis of the bowl. Besides, two different structures were found when the anions are located over the convex face (out), difering in the position of the anion in an outer or inner position. Thus out-2 presents the anion nearer the edge of the bowl whereas in out-1 the anion interacts with the carbon atoms of the central pentagon.

Table 2. Distances $(\AA)$ from the ion to the nearest carbon atom in the complexes.

\begin{tabular}{|l|l|c|c|c|c|c|c|}
\hline & & Cl5 & F5 & CN5 & Cl10 & F10 & CN10 \\
\hline $\mathbf{C l}^{-}$ & out-1 & 2.808 & 2.889 & 2.556 & 2.838 & 2.796 & 2.395 \\
\hline & out-2 & 3.211 & 3.044 & 2.978 & 2.879 & 2.800 & 2.608 \\
\hline & in & 3.180 & 3.204 & 3.136 & 3.075 & 3.121 & 3.113 \\
\hline Br $^{-}$ & out-1 & 3.029 & 3.092 & 2.778 & 3.057 & 3.007 & 2.656 \\
\hline & out-2 & 3.359 & 3.246 & 3.153 & 3.079 & 2.999 & 2.835 \\
\hline & in & 3.325 & 3.346 & 3.284 & 3.230 & 3.268 & 3.172 \\
\hline $\mathbf{B F}_{4}^{-}$ & out-1 & 3.100 & 3.114 & 3.050 & 3.154 & 3.082 & 3.032 \\
\hline & out-2 & 3.203 & 3.212 & 3.158 & 3.313 & 3.241 & 3.232 \\
\hline & in & 3.776 & 3.834 & 3.693 & 3.442 & 3.594 & 3.447 \\
\hline
\end{tabular}

Table 2 lists the distances from the anion to the nearest carbon atom of the bowl in the complexes formed with the different bowls. It can be observed that the distances follow the order of electrostatic potential, being shorter as the MEP becomes more positive, and therefore the interaction is more intense.

As expected, intermolecular distances are shorter in complexes formed by the convex face, since there is greater esteric hindrance by the concave side of the bowl, where atoms are closer. There is an anomalous behaviour corresponding to complexes formed with Coran-C110, presenting distances even shorter than for the other decasubstituted bowls. This is a consequence of the greater planarity of Coran-C110, thus allowing for anions to be closer to the bottom of the bowl. 

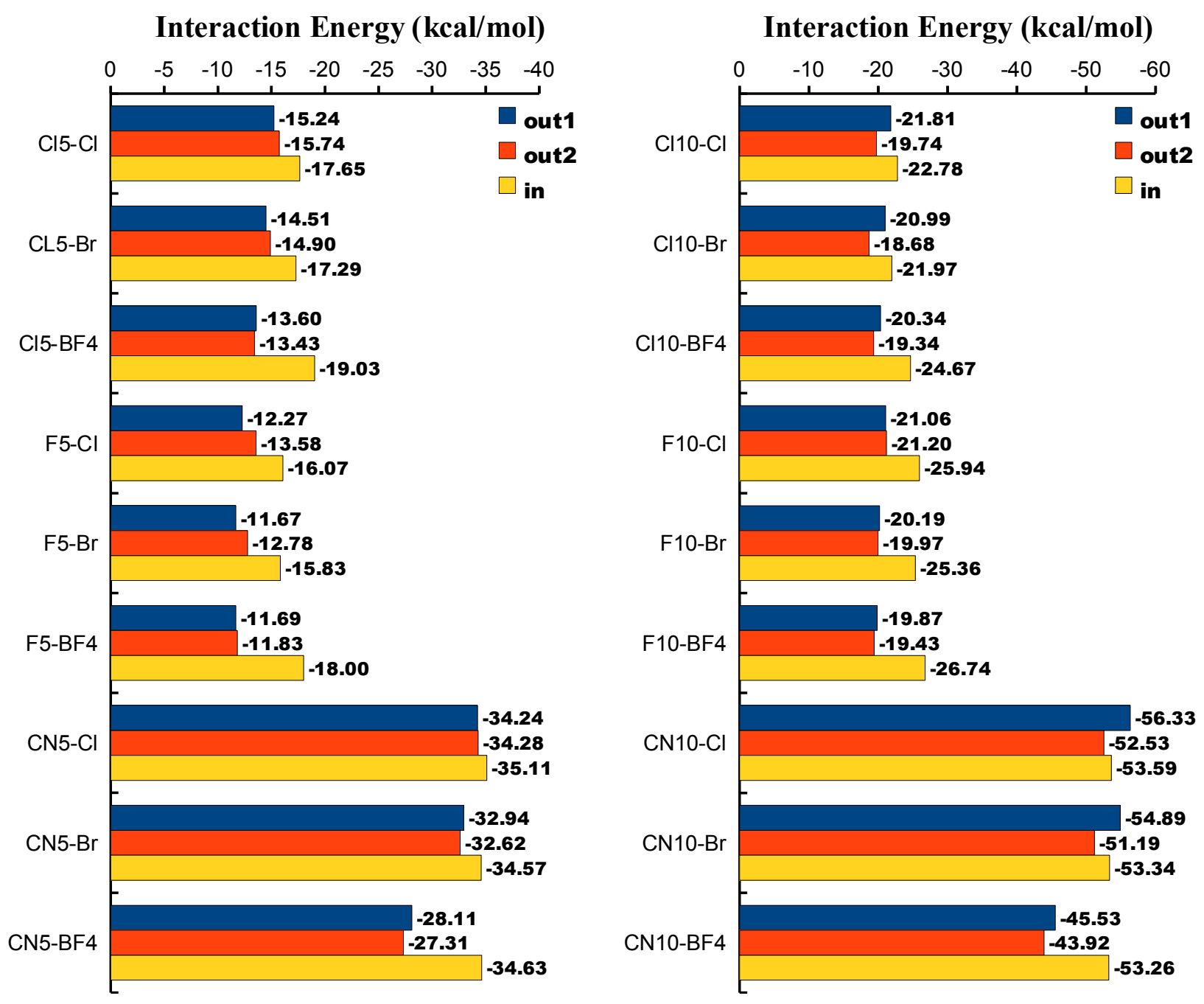

Figure 5. Interaction energies of the complexes studied in this work as obtained at the BLYP-D/aug-cc-pVDZ level of calculation 
Figure 5 shows the interaction energies calculated for the complexes between the anions and molecular bowls considered in this study, as obtained at the BLYP-D/aug-cc-pVDZ level.

Beginning with the corannulenes with five substituents, it can be observed that all anions form pretty stable complexes, the interaction energies amounting to -15 to $-20 \mathrm{kcal} / \mathrm{mol}$ for the bowls with chlorine and fluorine. The interaction energies are much larger, reaching $-35 \mathrm{kcal} / \mathrm{mol}$, for the complexes formed with Coran-CN5, as expected taking into account the larger electrostatic potential as shown in Figures 1 and 2.

Considering the different anions, it can be observed that the stabilities roughly follow the order $\mathrm{Cl}^{-}>\mathrm{Br}^{-}>\mathrm{BF}_{4}^{-}$, correlating with the polarizing power of the anion, and theferore with the stregth of the electrostatic interaction. However, it is worth noting that the complexes formed with $\mathrm{BF}_{4}^{-}$are as stable as those formed with $\mathrm{Cl}^{-}$or $\mathrm{Br}^{-}$in the out positions, and even more stable when the in structures are considered. The larger stability of complexes formed with the least polarizing anions suggests that other contributions as dispersion should play a major role stabilising these complexes, overcoming the less favorable electrostatic interaction, which is stronger in complexes formed with $\mathrm{Cl}^{-}$or $\mathrm{Br}^{-}$.

Moreover, the two complexes considered in this work with the convex side of the bowl present similar interaction energies in all cases, usually favoring the out-2 position in the pentasubstituted bowls whereas in the decasubstituted bowls the out-1 structure is more favored. This is probably due to the possibility of interaction with the rim hydrogen atoms in the pentasubstituted bowls, thus favoring a disposition of the anion nearer to the bowl edge. In any case, in most cases the most stable complex corresponds to the in structure.

To analyse in more detail the balance of energy contributions to the stability of the complexes, the interaction energy was decomposed in the two contributions of the model employed; that is, the pure BLYP interaction energy and the empirical dispersion contribution to the interaction energy. The results are shown in Figure 6. 

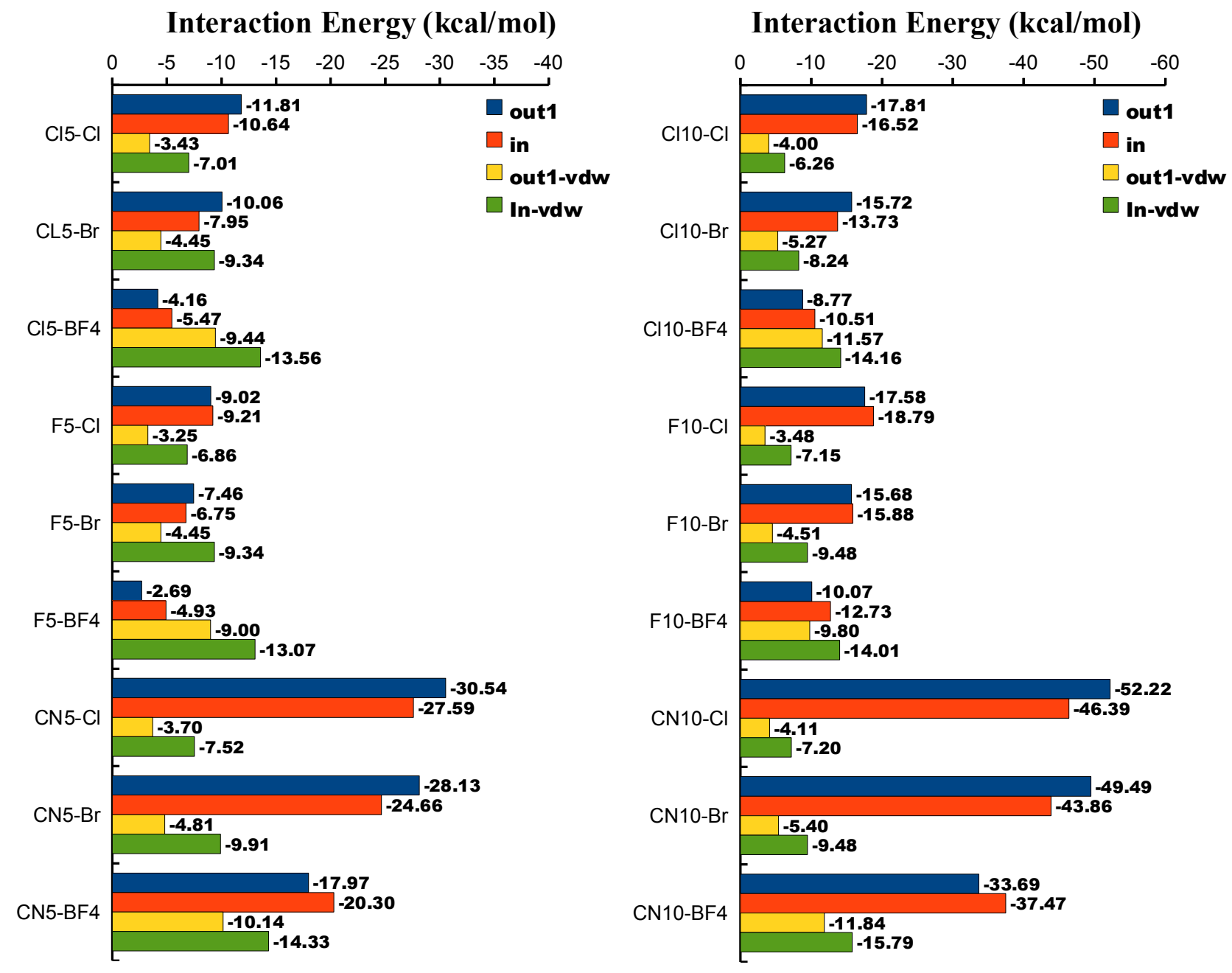

Figure 6. BLYP interaction energies and dispersion contributions of the complexes studied in this work as obtained at the BLYP-D/aug-cc-pVDZ level of calculation. 
It can be observed that for the pentasubstituted bowls, considering only the BLYP contribution, the complexes formed with the convex face are more stable than those formed with the concave side of the bowl. The exception are the complexes formed with $\mathrm{BF}_{4}^{-}$, for which the concave complex is more stable.

Though for the decasubstituted bowls the BLYP values are already more negative for the concave complexes, the dispersion contribution still favors the concave side of the bowl, so making the energy difference between the two sides even larger. What becomes clear from Figure 6 is that dispersion plays a major role in determining the order of stability of the complexes. As observed, complexes formed by the concave side always present larger dispersion componet due to the greater proximity of the atoms of the bowl.

So, we can conclude that including dispersion in the calculations is crucial for obtaining a correct stability ordering of the complexes, in contrast with cation complexes, where a simple DFT treatment with common functionals seems to suffice for getting a proper description of the interaction.

This is specially evident in complexes formed with more complex anions, as observed for the $\mathrm{BF}_{4}^{-}$complexes, with a quite small electrostatic interaction. Usually, the BLYP contribution in complexes formed with $\mathrm{BF}_{4}^{-}$is small, contributing to the stabilization of the complex even less than dispersion. This is the case of the complexes formed with the bowls with less positive molecular electrostatic potential, as Coran-C15 and Coran-F5, where the interaction is clearly dispersioncontrolled. As the MEP becomes more positive, the dispersion contribution decreases in relative terms, though for Coran-C110 and Coran-F10 still contributes with half of the total interaction energy of the complexes. 


\section{References}

(1) Hobza, P.; Zaradnik, R. Intermolecular complexes : the role of van der Waals systems in physical chemistry and the biodisciplines; Elsevier: Amsterdam, 1988.

(2) Meyer, E. A.; Castellano, R. K.; Diederich, F. Angew. Chem., Int. Ed. 2003, 42, 1210.

(3) Tsuzuki, S.; Uchimaru, T. Current Organic Chemistry 2006, 10, 745.

(4) Sherrill, C. D. Reviews in computational chemistry 2009, 26, 1.

(5) B. L. Schottel; Chifotides, H. T.; Dunbar, K. R. Chem. Soc. Rev. 2008, 37, 68.

(6) Klarner, F.-G.; Panitzky, J.; Preda, D.; Scott, L. T. Journal of Molecular Modeling 2000, 6,318 .

(7) Dunbar, R. C. J. Phys. Chem. A 2002, 106, 9809.

(8) Wu, Y.-T.; Siegel, J. S. Chem. Rev. 2006, 106, 4843.

(9) Garau, C.; Frontera, A.; Quiñonero, D.; Ballester, P.; Costa, A.; Deyá, P. M. J. Phys. Chem. A 2004, 108, 9423.

(10) Garau, C.; Frontera, A.; Quiñonero, D.; Ballester, P.; Costa, A.; Deyá, P. M. Chem. Phys. Lett. 2004, 392, 85.

(11) Berryman, O. B.; V. S. Bryantsev; D. P. Stay; Johnson, D. W.; Hay, B. P. J. Am. Chem. Soc. 2007, 129, 48.

(12) Hermida-Ramón, J. M.; Estévez, C. M. Chem. Eur. J. 2007, 13, 4743.

(13) Grimme, S. J. Comput. Chem. 2004, 25, 1463.

(14) Grimme, S. J. Comput. Chem. 2006, 27, 1787.

(15) Zhao, Y.; Truhlar, D. G. J. Chem. Theor. Comput. 2005, 1, 415.

(16) Zhao, Y.; Truhlar, D. G. J. Chem. Theory Comput. 2007, 3, 289.

(17) Antony, J.; Grimme, S. J. Phys. Chem. A 2007, 111, 4862.

(18) Grimme, S. J. Chem. Phys. 2003, 118, 9095.

(19) Peverati, R.; Baldridge, K. K. J. Chem. Theory Comput. 2008, 4, 2030.

(20) Boys, S. F.; Bernardi, F. Mol. Phys. 1970, 18, 553.

(21) Chalasinski, G.; Szczesniak, M. M. Chem. Rev. 2000, 100, 4227.

(22) Ahlrichs, R.; Bär, M.; Häser, M.; Horn, H.; Kölmel, C. Chem. Phys. Lett. 1989, 162, 165.

(23) Neese, F. ORCA. An Ab initio, DFT, and Semiempirical Electronic Structure Package, Version 2.6-35, University of Bonn, Germany (2007). 